\title{
Psoriasis: epidemiology, natural history, and differential diagnosis
}

\author{
This article was published in the following Dove Press journal: \\ Psoriasis: Targets and Therapy \\ 10 September 2012 \\ Number of times this article has been viewed
}

\section{Juliana L Basko-PIluska \\ Vesna Petronic-Rosic}

Department of Medicine, Section of Dermatology, University of Chicago, Chicago, IL, USA
Correspondence: Vesna Petronic-Rosic University of Chicago, Section of Dermatology, 584I S Maryland Ave, MC 5067, Chicago, IL 60637, USA

Tel +I 7737026559

Fax +I 7737023898

Email vrosic@medicine.bsd.uchicago.edu
Abstract: Psoriasis is a chronic, immune-mediated, inflammatory disease which affects primarily the skin and joints. It occurs worldwide, but its prevalence varies considerably between different regions of the world. Genetic susceptibility as well as environmental factors play an important role in determining the development and prognosis of psoriasis. Genomewide association studies have identified many genetic loci as potential psoriasis susceptibility regions, including PSORS1 through PSORS7. Histocompatibility antigen (HLA) studies have also identified several HLA antigens, with HLA-Cw6 being the most frequently associated antigen. Epidemiological studies identified several modifiable risk factors that may predispose individuals to developing psoriasis or exacerbate pre-existing disease. These include smoking, obesity, alcohol consumption, diet, infections, medications and stressful life events. The exact mechanism by which they trigger psoriasis remains to be elucidated; however, existing data suggest that they are linked through Th1-mediated immunological pathways. The natural history of psoriasis varies depending on the clinical subtype as well as special circumstances, including pregnancy and HIV infection. In general, psoriasis is a chronic disease with intermittent remissions and exacerbations. The differential diagnosis is vast and includes many other immunemediated, inflammatory disorders.

Keywords: psoriasis, epidemiology, natural history, differential diagnosis

\section{Epidemiology}

\section{Prevalence and incidence}

Psoriasis is a chronic, immune-mediated, inflammatory disease which primarily affects the skin and joints. The etiology of psoriasis is multifactorial and involves a complex interplay between genetic and environmental factors. Psoriasis occurs worldwide and has no gender preference; however, its prevalence varies considerably. In the US, psoriasis affects approximately $2 \%$ of the population, although rates as high as $4.6 \%$ have been reported. ${ }^{1,2}$ Bell et al showed an age- and sex-adjusted annual incidence of 60.4 in 100,000 for Rochester, MN, between the period of 1980 to $1983 .{ }^{3}$ More recently, Huerta et al reported an incidence rate of 14 per 10,000 person-years. ${ }^{4}$ The difference in reported incidence rate can be attributed to the different case definitions used in these studies. Psoriasis is extremely rare to absent in certain ethnic groups, such as Africans, African-Americans, Japanese, Alaskans, Australians, and Norwegian Lapps. ${ }^{2,5,6}$ The prevalence of psoriasis has not changed with time according to epidemiological studies, in contrast to other autoimmune diseases whose prevalence rates have increased. 


\section{Age of onset}

Psoriasis can first appear at any age; however, a bimodal distribution of the age of onset is characteristic. The majority of cases, approximately $75 \%$, present before the age of 40 years, with a peak at 20-30 years old. The remaining cases present after the age of 40 years. Patients with early disease onset tend to have a positive family history of psoriasis, frequent association with histocompatibility antigen (HLA)Cw6, and more severe disease. Those with onset after the age of 40 usually have a negative family history and a normal frequency of the $C w 6$ allele.

\section{Genetic basis}

The genetic basis of psoriasis is complex as multiple genes are involved. Based on twin studies, the heritability of psoriasis has been estimated to be $60 \%-90 \%$, which is among the highest of all multifactorial genetic diseases. ${ }^{7,8}$ Concordance rates as high as $70 \%$ have been reported among monozygotic twins, versus $12 \%-30 \%$ in dizygotic twins. ${ }^{9}$ Family studies indicate that if both parents have psoriasis, the offspring have a $50 \%$ chance of developing the disease. The risk decreases to $16 \%$ if only one parent has psoriasis. If neither parent is affected but a child develops psoriasis then their siblings have an $8 \%$ risk of developing the disease. Men have a higher risk of transmitting psoriasis to offspring than women, which is likely due to genomic imprinting. ${ }^{10}$ Genome-wide association studies have demonstrated seven major psoriasis susceptibility loci. A major susceptibility locus for psoriasis is located on chromosome 6p21 and is referred to as PSORS1. This region is believed to account for $35 \%-50 \%$ of psoriasis heritability. An association between psoriasis and other loci has also been reported on chromosomes 1p (PSORS7), 1q (PSORS4), 3q (PSORS5), 4q (PSORS3), 17q (PSORS2), and 19p (PSORS6). The strength of associations between such genes and susceptibility to psoriasis, apart from PSORS1, varies per study. HLA studies have shown that psoriasis is associated with several HLA antigens, most frequently HLACw6, which confers a relative risk of 10 for developing the disease in the Caucasian population. However, only approximately $10 \%$ of individuals who express the $H L A-C w 6$ allele go on to develop psoriasis. ${ }^{11}$ In addition, $H L A-C w 6$ influences the age of onset of the disease. HLA-Cw6 is expressed in about $85 \%-90 \%$ of patients with early-onset psoriasis, but in only $15 \%$ of patients with late-onset disease. ${ }^{7,12}$ HLAB13, HLA-B17, HLA-B37, and HLA-Bw16 have also been associated with plaque psoriasis. HLA-B27 is expressed with an increased frequency in pustular psoriasis and acrodermatitis continua of Hallopeau. A significant association between guttate psoriasis in children and HLA-B13 and HLA-B17 expression has been reported. These same HLA antigens are frequently expressed in erythrodermic psoriasis. Non-MHC genes, such as PTPN22, may also play a role in the development of psoriasis. PTPN22 encodes lymphoid protein tyrosine phosphatase, an enzyme which plays a role in the negative regulation of $\mathrm{T}-$ and $\mathrm{B}$-cell signaling. PTPN22 and its association to psoriasis have been extensively examined. A recent meta-analysis of ten studies showed a non-significantly positive association between psoriasis and the PTPN22. ${ }^{13}$ Despite numerous advances, understanding the genetic basis of psoriasis remains challenging and genetic testing is currently not clinically useful.

\section{Risk factors}

In addition to genetic susceptibility, environmental risk factors are implicated in triggering or exacerbating psoriasis (Table 1). Data from analytic epidemiologic studies (eg, case-control and nested cohort studies) with appropriate control for confounding variables have identified multiple risk factors: smoking, obesity, alcohol consumption, diet, infections, medications, and stressful life events. ${ }^{1,3,14-20}$ One of the largest studies investigating the association of smoking and obesity with psoriasis was the Nurses' Health Study II. This study, which included a cohort of over 78,000 nurses from the US, demonstrated a "dose-response" relationship for obesity and smoking on the risk of developing incident psoriasis..$^{21,22}$ Specifically, the study estimated that $30 \%$ of new psoriasis cases were due to being overweight (body mass index $>25$ ). European studies have also confirmed that current smoking and obesity are independent risk factors for developing psoriasis. ${ }^{14} \mathrm{~A}$ higher prevalence of psoriasis has been demonstrated in current smokers than never- or ex-smokers. ${ }^{23}$ Compared with patients who had never smoked, the multivariate relative risk of developing psoriasis was 1.78 for current smokers and 1.37 for past smokers $(P<0.05)$. For both current and past smokers, increased number of pack-years and increased intensity of smoking were both associated with graded increases in

Table I Psoriasis risk factors

\begin{tabular}{ll}
\hline Risk factor & References \\
\hline Smoking & $|4,2|-26$ \\
Obesity & $2 \mid$ \\
Alcohol consumption & $|6,29-3|$ \\
Infections & 36,37 \\
Medications & $39-43$ \\
Stressful life events & $1,36,45-46$ \\
\hline
\end{tabular}


the risk of psoriasis. Epidemiologic data and case-control studies from European populations have since confirmed the association between smoking and incident psoriasis and shown exposure to second-hand smoke at home to be a risk factor for psoriasis. ${ }^{24,25}$ A recent hospital-based crosssectional study of 818 adults with psoriasis showed that high intensity of smoking ( $>20$ cigarettes daily) versus a lower level of consumption ( $<10$ cigarettes daily) was associated with a greater than twofold increased risk of clinically more severe psoriasis. ${ }^{26}$ Cigarette-years significantly increased the risk of clinically more severe psoriasis after adjustment for confounding factors; the effect was stronger for women than men. Both smoking and obesity trigger Th-1 mediated immunological pathways, suggesting a plausible biologic explanation for these associations. ${ }^{27}$ Despite these data, it is not clear whether maintaining ideal body weight and avoiding smoking truly lowers one's risk of developing psoriasis. An Italian case-control study showed that nutritional habits, such as consumption of fruit and vegetables (carrots, tomatoes) or $\beta$-carotene are associated with a lower risk of psoriasis; however, this remains to be confirmed. ${ }^{28}$

Several large epidemiological studies have shown a significant correlation between alcohol consumption and psoriasis. A case-control study of 144 patients with psoriasis versus 285 unmatched controls with other skin diseases demonstrated that the odds ratio for developing psoriasis at an alcohol intake of $100 \mathrm{~g}$ per day compared to no intake was 2.2. ${ }^{16}$ A detailed survey by Higgins et al regarding drinking habits showed that the odds ratio for alcohol as an independent risk factor for psoriasis was 8.01. ${ }^{29}$ Most recently, Qureshi et al prospectively evaluated the association between total alcohol consumption and risk of incident psoriasis in a cohort of 82,869 nurses with no history of psoriasis. ${ }^{30}$ Compared with women who did not drink alcohol, the multivariate relative risk of psoriasis was 1.72 for an alcohol consumption of 2.3 drinks per week or more. When examined by type of alcoholic beverage, there was an association between psoriasis and non-light beer intake. Other alcoholic beverages did not increase the risk of incident psoriasis. This raises the question of whether the association of incident psoriasis is due to alcohol intake or due to a nonalcoholic component of non-light beer. Recently, a metaanalysis of case-control studies showed that the overall odds ratio of psoriasis for drinking persons compared to those with non-drinking habits was $1.531(P=0.002)$, suggesting that alcohol consumption is associated with an increased risk of psoriasis. ${ }^{31}$ However, the connection between psoriasis and alcohol consumption remains complex and has yet to be fully elucidated. Several other studies have not shown a significant correlation between alcohol consumption and psoriasis. ${ }^{16,21,26}$ The discrepancies are likely due to a non-standardized way of measuring alcohol consumption and patient recall bias. Finally, the influence of increasing alcohol consumption on the severity of psoriasis has also been investigated. Many studies suggest that heavy drinkers have a tendency to develop more extensive and inflamed skin lesions. ${ }^{16,20,32}$ Several mechanisms by which alcohol may induce psoriasis have been proposed, including upregulation of pro-inflammatory cytokines in various cell types, proliferation and activation of lymphocytes, hyperproliferation of keratinocytes as well as an increased risk of infection which can precipitate psoriasis. ${ }^{31,33-35}$

Acute bacterial and viral infections have been associated with the onset or exacerbation of psoriasis. ${ }^{36,37}$ Streptococcal infections are often triggers of guttate psoriasis, especially in children and young adults. ${ }^{38}$ Human immunodeficiency virus (HIV) has been associated with the onset of severe plaque psoriasis which is often non-responsive to standard treatment. Medications, including beta-blockers, lithium, antimalarials, tetracycline antibiotics, nonsteroidal anti-inflammatory drugs (NSAIDs), and steroid withdrawal have been associated with the onset or exacerbation of psoriasis, mostly based in case reports. ${ }^{39-42}$ Drug-induced psoriasis tends to occur in a de novo fashion in patients with no previous history of psoriasis, and usually resolve after discontinuation of the causative drug. Drug-aggravated psoriasis tends to occur in patients with a history of psoriasis and progresses even after discontinuation of the drug. The mechanism by which drugs provoke psoriasis is not well understood. Data suggest that beta blocker-induced psoriasis occurs as a result of epidermal $\beta 2$ receptor blockade leading to a decrease in intra-epidermal cyclic adenosine monophosphate (cAMP) and keratinocyte hyperproliferation. ${ }^{39}$ Many theories exist to explain the pathogenesis of lithium-provoked psoriasis. The current thinking is that lithium inhibits inositol monophosphate, causing depletion of intracellular calcium and increased keratinocyte proliferation. ${ }^{42}$ In addition, lithium may increase the production of tumor necrosis factor-alpha (TNF- $\alpha$ ) and interferon-gamma in keratinocytes, both key players in psoriasis. ${ }^{43}$ Antimalarial drugs may exacerbate pre-existing psoriasis in up to $40 \%$ of patients by inhibiting the enzyme transglutaminase and causing epidermal proliferation. ${ }^{43}$ The clinical relevance of antibiotic-provoked psoriasis remains controversial. The class of tetracyclines may exacerbate psoriasis through depletion of intracellular cAMP; however, this remains largely hypothetical. ${ }^{4}$ Finally, NSAIDs inhibit the 
metabolism of arachidonic acid, leading to an accumulation of leukotrienes, which are thought to aggravate psoriasis. Knowledge of the drugs that induce or exacerbate psoriasis can be of great importance in preventing precipitation or treating the disease.

Psychological stress has also been shown to trigger or exacerbate psoriasis. ${ }^{1,36,44,45}$ Up to $60 \%$ of patients describe stress as being a key "exacerbator" or trigger of their disease. Retrospective data have demonstrated that patients with psoriasis report more frequent traumatic experiences from early childhood to adulthood. Although most of the initial arguments for a link between stress and psoriasis came from anecdotal stories, recent studies in psychoneuroimmunology suggest that this link may exist. In addition to regulating the immune response, there is emerging evidence that abnormal neuroendocrine responses to stress may contribute to the pathogenesis of chronic autoimmune diseases, including psoriasis. In an experimental study of the effects of acute psychosocial stressors on 40 patients with psoriasis and 40 age-matched controls, altered hypothalamicpituitary-adrenal (HPA) responses were seen in patients with psoriasis - particularly those who experienced that their disease flared with stress. ${ }^{46}$ However, the relationship between stress and psoriasis is likely more complicated. A similar study of 24 psoriasis patients and 24 age-matched controls did not show the same positive correlation. ${ }^{47}$

\section{Natural history}

The natural history of psoriasis varies according to the clinical subtype. Psoriasis may present as chronic, stable plaques with intermittent remissions and exacerbations, or acutely with a rapid progression and widespread involvement. Plaque psoriasis is usually chronic with intermittent remissions. Plaques may persist for months to years at the same locations; however, periods of complete remission do occur. Remissions of 5 years or more have been reported in approximately $5 \%$ of patients. Plaque psoriasis responds well to topical therapy and phototherapy; however, systemic therapy may be necessary for severe disease. In addition to cutaneous involvement, plaque psoriasis may be associated with internal involvement, including joints and extra-articular sites such as the eyes. Concomitant psoriatic arthritis occurs in $5 \%-30 \%$ of patients with cutaneous psoriasis. ${ }^{5}$ In a minority of patients, the symptoms of psoriatic arthritis appear before skin involvement. The typical presentation is that of an asymmetric oligoarthritis with involvement of the distal (DIPs) and proximal (PIPs) interphalangeal joints of the hands and feet. Erosive changes may occur years after the presenting periarticular inflammation. The prevalence of ophthalmic involvement in patients with cutaneous disease is not known; however, it is thought to occur in approximately $10 \%$ of patients. ${ }^{48}$ Psoriasis may affect almost any part of the eye, leading to blepharitis, peripheral keratopathy, acute anterior uveitis, posterior synechiae, conjunctivitis, and cataract formation.

The prognosis of guttate psoriasis is excellent in children with spontaneous remissions occurring over the course of weeks to months. In adults, the lesions of guttate psoriasis may become chronic and progress to widespread involvement with plaque psoriasis. Palmoplantar pustulosis, which presents with sterile pustules of the palmoplantar surfaces admixed with yellow-brown macules, is a chronic disease which tends to remain localized to the palms and soles. This variant of psoriasis is less responsive to standard treatment and is commonly associated with sterile inflammatory bone lesions, such as SAPHO syndrome (synovitis, acne, pustulosis, hyperostosis, and osteitis). Furthermore, palmoplantar pustulosis is significantly aggravated by extrinsic factors, such as stress, smoking, and infections.

Generalized pustular psoriasis (von Zumbusch) is a severe form of psoriasis which is triggered by pregnancy, rapid withdrawal of corticosteroids, infections, and hypocalcemia. It is complicated by systemic symptoms of fever, chills, and fatigue, as well as electrolyte derangements and liver abnormalities. This variant requires systemic immunosuppressive therapy and often responds by resolution of pustules and extensive scaling after several days of treatment. The mortality rate due to sepsis is high without appropriate treatment.

In addition to the clinical subtype of psoriasis, pregnancy and HIV infection strongly influence the natural course of disease.

\section{Psoriasis and pregnancy}

It is well established that psoriasis fluctuates during pregnancy, likely due to the hormonal changes in estrogen and progesterone resulting in a state of altered immune surveillance. The majority of women usually experience an improvement in their cutaneous disease during pregnancy. According to a study by Murase et al, 55\% of the patients reported improvement during pregnancy, 21\% no change, and $23 \%$ reported worsening. ${ }^{49}$ However, only $9 \%$ of patients reported improvement, $26 \%$ no change, and 65\% reported worsening postpartum. In patients with $10 \%$ or greater body surface area involvement who reported improvement, lesions decreased by $83.8 \%$ during pregnancy. Similar data were obtained by Raychaudhuri et al. ${ }^{50}$ Of the 91 pregnant 
women involved in the study, 51 (56\%) improved, 24 (26.4\%) worsened, and $16(17.6 \%)$ had no change during pregnancy. Relapse during the early postpartum period was common. The mechanism by which psoriasis tends to improve during pregnancy is not well understood. However, there is now data to suggest that the upregulation of Th2 cytokines during pregnancy counteracts the effects of pro-inflammatory Th1 cytokines which are key players in the pathogenesis of psoriasis.

\section{Psoriasis and HIV}

HIV-associated psoriasis occurs with a similar to slightly higher prevalence than in the general population. ${ }^{51-53}$ Psoriasis may present for the first time in advanced HIV infection, when CD4-cell count is less than 100 cells per $\mu \mathrm{L},{ }^{54}$ and could even be the initial clinical manifestation of HIV infection. ${ }^{53}$ However, psoriasis also tends to spontaneously improve during end-stage AIDS. ${ }^{52,55}$ The relationship between HIV infection and psoriasis is complicated. While an expansion of CD8 cells in advanced HIV might explain the exacerbation of psoriasis, ${ }^{56}$ there is now evidence that psoriasis patients are enriched for genetic variants that protect against HIV-1 disease. ${ }^{57}$ Further studies are needed to shed light on this association.

\section{Psoriasis and its linkage to other diseases}

Patients with psoriasis may be at increased risk of developing other diseases due to shared genetic pathways, common immune mechanisms, treatment-related toxicities, and the associated psychological burden of the disease (Table 2). Psoriasis patients have an increased prevalence of inflammatory bowel disease, especially Crohn's disease, which may be due to shared genetic loci. PSORS8 has been shown to overlap with a Crohn's disease locus on chromosome 16q. ${ }^{58}$ Furthermore, both Crohn's disease and psoriasis are Th- 1 mediated conditions and are characterized by elevated levels of the pro-inflammatory cytokine TNF- $\alpha$, which is central to the pathogenesis of both conditions. Chronic Th-1 inflammation

Table 2 Psoriasis and its linkage to other diseases

\begin{tabular}{ll}
\hline Linkage to other diseases & References \\
\hline Inflammatory bowel disease (especially Crohn's) & 58 \\
Obesity & $14,27,59-64,66,67$ \\
Metabolic syndrome & 68 \\
Type II diabetes & 65 \\
Cardiovascular disease & 66,69 \\
Malignancy & $73-77,79-8 \mid$ \\
Psychiatric comorbidities & $82-88$ \\
\hline
\end{tabular}

is also implicated in the pathogenesis of a diverse group of conditions such as insulin resistance, atherosclerosis, and thrombosis, all of which may be more frequently encountered in psoriasis patients. ${ }^{60}$ Epidemiological studies have shown that patients with psoriasis have a higher risk of developing certain metabolic disorders, particularly obesity. ${ }^{61-64}$ Psoriasis and obesity may be linked through a common pathophysiological mechanism of chronic low-grade inflammation. In an Italian case-control study with 560 psoriatic patients, the odds of having psoriasis with a body mass index between 26 and 29 or above 30 were 1.6 and 1.9, respectively, compared to non-obese control subjects. ${ }^{14}$ A study from the UK of 127,706 patients with mild psoriasis and 3854 patients with severe psoriasis demonstrated higher adjusted odds of obesity in patients with severe psoriasis $(\mathrm{OR}=1.8)$ than in patients with mild psoriasis $(\mathrm{OR}=1.3)$ compared with patients without psoriasis. ${ }^{27}$ Several mechanisms by which psoriasis could lead to obesity have been proposed, including increased social isolation, unhealthy dietary habits, increased depression, alcohol consumption, and decreased physical activity. The question of whether psoriasis predisposes to obesity or whether obesity is a risk factor for the development of psoriasis, however, remains debatable. There is also evidence that obesity predisposes individuals to the development of psoriasis. Prospective data on 78,626 women in the Nurses' Health Study II found that weight-gain placed individuals at an increased risk for the development of psoriasis. ${ }^{21}$ Obesity is a proinflammatory state and adipose tissue is a rich source of inflammatory mediators, such as adiponectin, leptin, resistin, TNF- $\alpha$, interleukin 6, and monocyte chemoattractant protein 1 . These products are thought to provide an important link between obesity, insulin resistance and psoriasis. The prevalence of diabetes and metabolic syndrome is also increased in psoriasis patients. ${ }^{27,61,63-65} \mathrm{~A}$ recent study demonstrated a higher prevalence of diabetes in patients with psoriasis independent of traditional diabetes risk factors such as age, gender, obesity, hypertension, and hyperlipidemia. ${ }^{65}$ Patients suffering from non-familial and late-onset psoriasis were found to have the highest risk. Contrary to what was found by Gelfand et al, ${ }^{66}$ patients with more severe disease did not have a higher risk of suffering from type 2 diabetes. The potential link between psoriasis and type 2 diabetes is not well established; however, the present data suggest that chronic inflammation in the setting of psoriasis may predispose to the development of insulin resistance and diabetes. Gisondi et al performed a hospital-based case-control study on 338 adult patients with chronic plaque psoriasis and 334 patients with skin diseases other than psoriasis. ${ }^{67}$ They demonstrated that psoriatic 
patients have a higher prevalence of metabolic syndrome (30.1\% vs $20.6 \%, P=0.005$ ), independent from smoking. In addition, psoriatic patients had a higher prevalence of hypertriglyceridemia and abdominal obesity. However, there was no correlation between severity of psoriasis and prevalence of metabolic syndrome.

Patients with psoriasis have increased rates of cardiovascular disease that are not accounted for by cardiovascular risk factors such as obesity, diabetes, hypertension, hyperlipidemia, or smoking. Recent case-control studies have demonstrated that patients with psoriasis have lower levels of folate and higher levels of homocysteine in comparison to normal controls. ${ }^{68,69}$ Hyperhomocysteinemia in psoriasis may be directly related to folate deficiency. Homocysteine is an independent risk factor for cardiovascular disease, which could explain why psoriatic patients have an increased risk of cardiovascular events independent of other risk factors. However, hyperhomocysteinemia could also be related to other risk factors such as hypertension, obesity, smoking, and excessive alcohol consumption, all of which have significant prevalence in psoriatic patient population. This could explain why lowering homocysteine levels has not been shown to reduce vascular outcomes. ${ }^{70}$ Ludwig et al demonstrated that the prevalence and severity of coronary artery disease was greater in patients with severe psoriasis than matched controls, even after controlling for major cardiovascular risk factors. ${ }^{71}$ Gelfand et al demonstrated in a large observational cohort study that patients with mild and severe psoriasis had an increased risk of myocardial infarction. ${ }^{66}$ The adjusted relative risk of myocardial infarction was greatest in younger patients with severe disease. These data suggest that psoriasis may be a marker of increased risk of myocardial infarction, especially in young patients. However, a more recent study by Fernandez-Torres et al looking at cardiovascular risk in 395 psoriatic patients using the different cardiovascular risk scores did not find a correlation between the severity and duration of psoriasis and cardiovascular risk, which shows the complexity of this relationship. ${ }^{72}$

The immunologic nature of psoriasis, as well as the immunosuppressive therapies used for treatment, may predispose patients to an increased risk of cancer. A higher incidence of non-melanoma skin cancer has been reported, whereas findings regarding internal solid cancers such as lung, breast, colon, and prostate are conflicting. ${ }^{73-76}$ A recent retrospective population-based cohort study by Chen et al showed that psoriasis carries an elevated risk of malignancy, especially in younger, male patients, independent of systemic treatment. ${ }^{77}$ Certain cancers were significantly associated with psoriasis, including those of the skin and urinary bladder, followed by oropharynx/larynx, liver/gallbladder, and colon/rectum. One proposed explanation for the increased cancer risk is that psoriatic patients have more common risk factors for malignancies, including a higher tobacco and alcohol consumption as well as unhealthy lifestyles. Studies of the risk of lymphoma in psoriasis patients have yielded inconsistent results. The largest study to date found no increased risk of non-Hodgkin's lymphoma, but showed an increased risk of Hodgkin's lymphoma and cutaneous T-cell lymphoma, especially in those 65 years or older. ${ }^{78}$ Multiple studies have examined the psychological comorbidities in patients with psoriasis. ${ }^{79-81}$ It is now well-established that the disease has a major impact on the patient's quality of life. ${ }^{82,83}$ A wide range of problems, including major depression, anxiety, obsessive behavior, sexual dysfunction, and suicidal ideation, have been described. ${ }^{84-87}$ A study comparing 50 patients with psoriasis in outpatient clinics to 50 healthy controls found that patients with psoriasis had a higher average depression score ${ }^{88}$ In addition, suicidal ideation was higher in patients with more severe disease. In another study of 217 patients, 9.7\% reported a wish to be dead and another 5.5\% reported active suicidal ideation. ${ }^{80}$ However, there are no data estimating the prevalence of completed suicides in patients with psoriasis. Contrary to popular belief, a recent review of the psychiatric implications of psoriasis by Rieder et al showed that the extent of psoriasis does not predict severity of psychosocial morbidity. ${ }^{89}$ Patients with psoriasis often continue to be distressed even after their cutaneous lesions have resolved. According to a recent prospective study of 414 hospital inpatients, one-third of patients with complete clearance of their psoriasis still presented psychosocial difficulties at followup. ${ }^{90}$

\section{Differential diagnosis}

The differential diagnosis of psoriasis is vast and depends on the clinical subtype (Table 3 ). Chronic plaque psoriasis

Table 3 Differential diagnoses of psoriasis

\begin{tabular}{ll}
\hline $\begin{array}{l}\text { Cutaneous T cell lymphoma/ } \\
\text { mycosis fungoides }\end{array}$ & Pityriasis rosea \\
\hline Pityriasis rubra pilaris & Pityriasis lichenoides chronica \\
Nummular dermatitis & Secondary syphilis \\
Bowen's disease & Acute generalized \\
& exanthematous pustulosis \\
Hypertrophic lichen planus & Sneddon-Wilkinson disease \\
Small plaque parapsoriasis & Langerhans cell histiocytosis \\
Intertrigo & Dyshidrotic dermatitis \\
Tinea manuum/pedum/capitis & Seborrheic dermatitis \\
\hline
\end{tabular}


must be distinguished from the mycosis fungoides variant of cutaneous T-cell lymphoma (CTCL). The latter often exhibits signs of epidermal atrophy or poikiloderma which allow its differentiation from plaque psoriasis; however, occasionally, a skin biopsy is the only way to distinguish between the two. Pityriasis rubra pilaris (PRP) is clinically distinguished from plaque psoriasis by the presence of red-orange palmoplantar keratoderma, keratotic follicular papules, and the classic islands of sparing on the trunk. Histopathologically, the alternating vertical and horizontal ortho- and parakeratosis as well as follicular plugging help distinguish PRP from psoriasis. Nummular dermatitis and Bowen's disease are also in the differential diagnosis of plaque psoriasis. Nummular dermatitis is more pruritic than psoriasis and is often associated with a history of atopy. Histopathological examination is the most reliable way to differentiate between a plaque of psoriasis and Bowen's disease. When psoriasis involves the shins, a diagnosis of hypertrophic lichen planus may be entertained, but typical lichen planus lesions elsewhere on the body as well as mucosal involvement may help to differentiate between the two. Guttate psoriasis is often straightforward to diagnose; however, the clinical differential diagnosis includes small plaque parapsoriasis, pityriasis rosea, pityriasis lichenoides chronica (PLC), and secondary syphilis. These entities can be distinguished on the basis of key historical, clinical, pathologic, and laboratory findings. Small plaque parapsoriasis usually presents with variably erythematous plaques which are covered with fine scale. Occasionally, it may present with elongated, finger-like patches symmetrically distributed on the flanks, also known as "digitate dermatosis." Pityriasis rosea is distinguished from psoriasis by the presence of a herald patch and resolution of the lesions within a few months. Secondary syphilis may be clinically distinguished on the basis of palmoplantar involvement, which does not happen in guttate psoriasis, as well as on the basis of histopathologic and serologic findings. PLC is clinically characterized by recurrent crops of spontaneously regressing, scaly red-brown papules. Histologically, there is an interface dermatitis composed predominantly of monoclonal T-lymphocytes and necrotic keratinocytes. Pustular psoriasis may resemble a pustular drug reaction, such as acute generalized exanthematous pustulosis (AGEP), Sneddon-Wilkinson disease, or IgA pemphigus. The presence of peripheral eosinophilia and tissue eosinophils on the skin biopsy, as well as a history of a preceding culprit medication favors AGEP. Sneddon-Wilkinson disease can be distinguished clinically by its annular or polycyclic plaques with a predilection for flexural surfaces. The diagnosis of IgA pemphigus is made via positive direct immunofluorescence, which is absent in both psoriasis and Sneddon-Wilkinson disease. The annular form of pustular psoriasis may also mimic Sneddon-Wilkinson disease. The principal differential diagnostic considerations of flexural psoriasis include intertrigo and Langerhans cell histiocytosis in infants. Patients with Langerhans cell histiocytosis may have scaling and crusting of the scalp, as well as internal organ involvement (eg, hepatomegaly, lytic bone lesions). A skin biopsy is diagnostic. The differential diagnoses for palmoplantar psoriasis include dyshidrotic dermatitis and tinea manuum/pedum. Yellow-brown macules intermixed with sterile pustules favor a diagnosis of palmoplantar psoriasis. $\mathrm{KOH}$ examination of the associated scale can help diagnose a dermatophyte infection. Nail psoriasis may mimic alopecia areata, lichen planus, or trachyonychia. Nail pitting is a feature of both psoriasis and alopecia areata; however, pits are large, deep and irregularly distributed in psoriasis in contrast to alopecia areata, where they are small, superficial, and regularly distributed. Oil drops, distal onycholysis, and splinter hemorrhages may also serve to distinguish between the two entities. Lateral nail thinning, longitudinal ridging, fissuring, and dorsal pterygium are features which favor lichen planus. Trachyonychia, or twenty-nail dystrophy, may be due to alopecia areata, lichen planus, or psoriasis. In the absence of cutaneous findings, a nail unit biopsy may help establish the underlying cause. Scalp psoriasis may resemble tinea capitis or seborrheic dermatitis. While psoriasis can often be clinically differentiated from tinea capitis, laboratory testing is sometimes necessary to establish the diagnosis of a dermatophyte infection. This includes a $\mathrm{KOH}$ preparation, a fungal culture, or microscopic examination of a skin biopsy specimen. Seborrheic dermatitis and psoriasis may present clinically very similarly and often respond to the same topical treatment. The presence of psoriatic plaques on the trunk as well as a positive family history may favor psoriasis. Histopathological examination remains the standard of differentiating between the two. Finally, erythrodermic psoriasis has a vast differential, as there are many other causes of erythroderma including seborrheic dermatitis, atopic dermatitis, Sezary syndrome, PRP, drug reactions, and graft-versus-host disease. A skin biopsy may or may not establish the underlying cause. Often, psoriatic erythroderma is preceded by classic psoriatic plaques; however, typical features of psoriasis are lost after generalization of the erythema. Nail changes, such as nail pitting, oil drops, or onycholysis may still be visible and provide a clue to the diagnosis of psoriatic erythroderma. 


\section{Conclusion}

Psoriasis is a chronic, multifactorial disorder which involves the interplay between a myriad of genetic and environmental risk factors. Based on the clinical subtype, psoriatic skin disease can have a variable clinical course and may mimic other inflammatory and autoimmune skin conditions. In addition to affecting the skin, psoriasis is associated with physical and emotional comorbidities which have a negative impact on the quality of life of the affected patients. Future studies are needed to determine if successful treatment of psoriasis will lead to a decreased risk of such comorbidities. Behavior modification and the use of preventative health screenings in patients with psoriasis may be useful until then.

\section{Disclosure}

The authors report no conflicts of interest in this work.

\section{References}

1. Naldi L. Epidemiology of psoriasis. Curr Drug Targets Inflamm Allergy. 2004;3:121-128.

2. Raychaudhuri SP, Farber EM. The prevalence of psoriasis in the world. J Eur Acad Dermatol Venereol. 2001;15:16-17.

3. Bell LM, Sedlack R, Beard CM, Perry HO, Michet CJ, Kurland LT. Incidence of psoriasis in Rochester, Minn, 1980-1983. Arch Dermatol. 1991;8:1184-1187.

4. Huerta C, Rivero E, Garcia Rodriguez LA. Incidence and risk factors for psoriasis in the general population. Arch Dermatol. 2007;143(12): 1559-1565.

5. Christophers E. Psoriais-epidemiology and clinical spectrum. Clin Exp Dermatol. 2001;26:314-320.

6. Gelfand JM, Stern RS, Nijsten T, et al. The prevalence of psoriasis in African Americans: results from a population-based study. $J$ Am Acad Dermatol. 2005;52(1):23-26.

7. Elder J, Nair R, Guo S, Henseler T, Christophers E, Voorhees J. The genetics of psoriasis. Arch Dermatol. 1994;130:216-224.

8. Elder JT, Nair RP, Henseler T, et al. The genetics of psoriasis 2001: the odyssey continues. Arch Dermatol. 2001;137:1447-1454.

9. Valdimarsson H. The genetic basis of psoriasis. Clin Dermatol. 2007; 25(6):563-567.

10. Rahman PEJ. Genetic epidemiology of psoriasis and psoriatic arthritis. Ann Rheum Dis. 2005;64(Suppl 2):ii37-ii39.

11. Trembath R, Clough RL, Rosbotham JL, et al. Identification of a major susceptibility locus on chromosome $6 \mathrm{p}$ and evidence for further disease loci revealed by a two stage genome-wide search in psoriasis. Hum Mol Genet. 1997;6:813-820.

12. Richardson SK, Gelfand J. Update on the natural history and systemic treatment of psoriasis. Adv Dermatol. 2008;24:171-196.

13. Chen YF, Chang J. PTPN2 C1858T and the risk of psoriasis: a meta-analysis. Mol Bio Rep. 2012;39:7861-7870.

14. Naldi L, Chatenoud L, Linder D, et al. Cigarette smoking, Body Mass Index, and stressful life events as risk factors for psoriasis: results from an Italian case-control study. J Invest Dermatol. 2005;125:61-67.

15. Naldi L, Parazzini F, Brevi A, et al. Family history, smoking habits, alcohol consumption and risk of psoriasis. $\mathrm{Br} J$ Dermatol. 1992;3:212-217.

16. Poikolainen K, Reunala T, Karvonen J, Lauharanta J, Karkkainen P. Alcohol intake: a risk factor for psoriasis in young and middle-aged men? BMJ. 1990;300:780-783.

17. Mills C, Srivastava E, Harvey I, et al. Smoking habits in psoriasis: a case control study. Br J Dermatol. 1992;127:18-21.
18. Duffy D, Spelman L, Martin N. Psoriasis in Australian twins. JAm Acad Dermatol. 1993;29:428-434.

19. Poikolainen K, Reunala T, Karvonen J. Smoking, alcohol and life events related to psoriasis among women. Br J Dermatol. 1994;130: 473-477.

20. Naldi L, Peli L, Parazzini F. Association of early-stage psoriasis with smoking and male alcohol consumption: evidence from an Italian casecontrol study. Arch Dermatol. 1999;135:1479-1484.

21. Setty AR, Curhan G, Choi HK. Obesity, wait circumference, weight change, and the risk of psoriasis in women: Nurses' Health Study II. Arch Intern Med. 2007;167(15):1670-1675.

22. Setty AR, Curhan G, Choi HK. Smoking and the risk of psoriasis in women: Nurses' Health Study II. Am J Med. 2007;120:953-959.

23. Schafer T. Epidemiology of psoriasis. Dermatology. 2006;212: 327-337.

24. Favato G. High incidence of smoking habit in psoriatic patients. Am J Med. 2008;121:e17.

25. Jankovic S, Raznatovic M, Marinkovic J, et al. Risk factors for psoriasis: a case-control study. J Dermatol. 2009;36:328-334.

26. Fortes C, Mastroeni S, Leffondre K, et al. Relationship between smoking and the clinical severity of psoriasis. Arch Dermatol. 2005;141:1580-1584

27. Neimann AL, Shin DB, Wang X, Margolis DJ, Troxel AB. Prevalence of cardiovascular risk factors in patients with psoriasis. $J$ Am Acad Dermatol. 2006;55(5):829-835.

28. Naldi L, Parazzini F, Peli L, Chatenoud L, Cainelli T. Dietary factors and the risk of psoriasis: results of an Italian case-control study. $\mathrm{Br} J$ Dermatol. 1996;134:101-106.

29. Higgins EM, Peters TJ, du Vivier AW. Smoking, drinking and psoriasis. Br J Dermatol. 1993;129:749-750.

30. Qureshi AA, Dominguez PL, Choi HK, Han J, Curhan G. Alcohol intake and risk of incident psoriasis in US women. Arch Dermatol. 2010;146(12):1364-1369.

31. Zhu KJ, Zhu CY, Fan YM. Alcohol consumption and psoriatic risk: A meta-analysis of case-control studies. J Dermatol. 2012; $39: 1-4$.

32. Higgins EM, du Vivier AW. Alcohol and the skin. Alcohol Alcohol. 1992;27:595-602.

33. Ockenfels HM, Keim-Maas C, Funk R, Nussbaum G, Goos M. Ethanol enhances the IFN-gamma, TGF-alpha and IL-6 secretion in psoriatic co-cultures. Br J Dermatol. 1996;135(5):746-751.

34. Farkas A, Kemeny L. Psoriasis and alcohol: is cutaneous ethanol one of the missing links? Br J Dermatol. 2010;162:711-716.

35. Farkas A, Kemény L, Széll M, Dobozy A, Bata-Csörgo Z. Ethanol and acetone stimulate the proliferation of $\mathrm{HaCaT}$ keratinocytes: the possible role of alcohol in exacerbating psoriasis. Arch Dermatol Res. 2003;295(2):56-62.

36. Naldi L, Peli L, Parazzini F, Carrel C; Dermatology PSGotIGERi. Family history of psoriasis, stressful life event, and recent infectious diseases are risk factors for a first episode of acute guttate psoriasis: results of a case-control study. J Am Acad Dermatol. 2001;44:433-438.

37. Krueger G, Duvic M. Epidemiology of psoriasis: clinical issues. J Invest Dermatol. 1994;102:14S-18S.

38. Zhao G, Feng X, Na A, et al. Acute guttate psoriasis patients have positive streptococcus hemolyticus throat cultures and elevated antistreptococcal M6 protein titers. J Dermatol. 2005;32:91-96.

39. Tsankov N, Irena A, Kasandjieva J. Drug-induced psoriasis: recognition and management. Am J Clin Dermatol. 2000;1:159-165.

40. Kim GK, Del Rosso JQ. Drug-provoked psoriasis: is it drug induced or drug aggravated? Understanding pathophysiology and clinical relevance. J Clin Aesthetic Dermatol. 2010;3(11):3-38.

41. Basavaraj KH, Ashok NM, Rashmi R, Praveen TK. The role of drugs in the induction and/or exacerbation of psoriasis. Int J Dermatol. 2010;49: 1351-1361.

42. O'Brian M, Koo J. The mechanism of lithium and beta blocker agents in inducing and exacerbating psoriasis. $J$ Drugs Dermatol. 2006;5: 426-433. 
43. Lionel F, Baker B. Triggering psoriasis: the role of infections and medications. Clin Dermatol. 2007;25:606-615.

44. Fortune DG, Richards HL, Griffiths C. Psychologic factors in psoriasis: consequences, mechanisms, interventions. Dermatol Clin. 2005;23:681-694.

45. Tausk F, Elenkov I, Moynihan J. Psychoneuroimmunology. Dermatol Ther. 2008;21:22-31.

46. Richards HL, Ray DW, Kirby B, et al. Response of the hypothalamicpituitary-adrenal axis to psychosocial stress in patients with psoriasis Br J Dermatol. 2005;153:1114-1120.

47. Karanikas E, Harsoulis F, Giouzepas I, et al. Neuroendocrine stimulatory tests of hypothalamic-pituitary-adrenal axis in psoriasis and correlative implications with psychopathological and immune parameters. $J$ Dermatol. 2009;36:35-44.

48. Rehal B, Modjtahedi BS, Morse LS, Schwab IR, Maibach HI. Ocular psoriasis. J Am Acad Dermatol. 2011;65:1202-1212.

49. Murasse JE, Chan KK, Garite TJ, Cooper DM, Weinstein GD. Hormonal effect on psoriasis in pregnancy and post partum. Arch Dermatol. 2005;141(5):601-606.

50. Raychaudhuri SP, Navare T, Gross J, Raychaudhuri SK. Clinical course of psoriasis during pregnancy. Int J Dermatol. 2003;42(7): $518-520$.

51. Wolfer LU, Djemadji-Oudjiel N, Hiletework M, et al. HIV-associated psoriasis. Clinical and histological observations in 36 patients. Hautarzt. 1998;49(3):197-202. German.

52. Obuch ML, Maurer TA, Becker B, Berger TG. Psoriasis and human immunodeficiency virus infection. J Am Acad Dermatol. 1992;27: 667-673.

53. Badger J, Berger TG, Gambla C, Koo JY. HIV and psoriasis. Clin Rev Allergy Immunol. 1996;14(4):417-431.

54. Munoz-Perez MA, Rodriguez-Pichardo A, Camacho F, Colmenero MA. Dermatological findings correlated with CD4 lymphocyte counts in a prospective 3 year study of 1161 patients with human immunodeficiency virus disease predominantly acquired through intravenous drug abuse. Br J Dermatol. 1998;139(1):33-39.

55. Colebunders R, Blot K, Meriens V, Dock P. Psoriasis regression in terminal AIDS. Lancet. 1992;339:1110.

56. Fife DJ, Waller JM, Jeffes EW, Koo JY. Unraveling the paradoxes of HIV-associated psoriasis: a review of T-cell subsets and cytokine profiles. Dermatol Online J. 2007;13:4.

57. Chen H, Hayashi G, Lai OY, et al. Psoriasis patients are enriched for genetic variants that protect against HIV-1 disease. PLoS Genet. 2012;8(2):e1002514

58. Karason AGJ, Upmanyu R, Antonsdottir AA, et al. A susceptibility gene for psoriatic arthritis maps to chromosome 16q: evidence for imprinting. Am J Hum Genet. 2003;72(1):125-131.

59. Lee FIBS, Francis C. Increased occurrence of psoriasis in patients with Crohn's disease and their relatives. AM J Gastroenterol. 1990;85 962-963

60. Gottlieb AB, Chao C, Dann F. Psoriasis comorbidities. J Dermatol Treat. 2008;19:5-21.

61. Cohen AD, Sherf M, Vidavsky L, Vardy DA, Shapiro J, Meyerovitch J. Association between psoriasis and the metabolic syndrome. A crosssectional study. Dermatology. 2008;216:152-155.

62. Mallbris L, Granath F, Hamsten A, Stahle M. Psoriasis is associated with lipid abnormalities at the onset of skin disease. J Am Acad Dermatol. 2006;54:614-621.

63. Sommer DM, Jenisch S, Suchan M, Christophers E, Weichenthal M. Increased prevalence of the metabolic syndrome in patients with moderate to severe psoriasis. Arch Dermatol Res. 2006;298:321-328.

64. Chen YJ, Wu CY, Shen JL, et al. Psoriasis independently associated with hyperleptinemia contributing to metabolic syndrome. Arch Dermatol. 2008;144:1571-1575.

65. Armesto S, Santos-Juanes J, Galache-Osuna C, Martinez-Camblor P, Coto E, Coto-Segura P. Psoriasis and type 2 diabetes risk among psoriatic patients in a Spanish population. Australas J Dermatol. 2012 53:128-130.
66. Gelfand JM, Neimann AL, Shin DB, Wang X, Margolis DJ, Troxel AB. Risk of myocardial infarction in patients with psoriasis. JAMA. 2006;296(14):1735-1741.

67. Gisondi P, Tessari A, Conti S, et al. Prevalence of metabolic syndrome in patients with psoriasis: a hospital-based case-control study. $\mathrm{Br} \mathrm{J}$ Dermatol. 2007;157:68-73.

68. Tobin AM, Hughes R, Hand EB, Leong T, Graham IM, Kirby B. Homocysteine status and cardiovascular risk factors in patients with psoriasis: a case-control study. Clin Exp Dermatol. 2011;36(1): 19-23.

69. Malerba M, Gisondi P, Radaeli A, Sala R, Calzavara P, Girolomoni G. Plasma homocysteine and folate levels in patients with chronic plaque psoriasis. Br J Dermatol. 2006;115(6):1165-1169.

70. Armitage JM, Bowman L, Clarke RJ, et al. Effects of homocysteinelowering with folic acid plus vitamin B12 versus placebo on mortality and morbidity in myocardial infarction survivors: a randomized trial. JAMA. 2010;303(24):2486-2494.

71. Ludwig RHC, Rostock A, Ochsendorf F. Psoriasis: a possible risk factor for development of coronary artery calcification. Br J Dermatol. 2006;156(2):271-276.

72. Fernandez-Torres, Pita-Fernandez, Fonseca E. Psoriasis and cardiovascular risk. Assessment by different cardiovascular risk scores. J Eur Acad Dermatol Venereol. 2012. [Epub ahead of print.]

73. Frentz G, Olsen JH, Avrach WW. Malignant tumors and psoriasis: climatotherapy at the Dead Sea. Br J Dermatol. 1999;141(6): 1088-1091.

74. Boffetta P, Gridley G, Lindelof B. Cancer risk in a population-based cohort of patients hospitalized for psoriasis in Sweden. J Invest Dermatol. 2001;117(6):1531-1537.

75. Hannuksela-Svahn A, Pukkala E, Laara E, Poikolainen K, Karvonen J. Psoriasis, its treatment, and cancer in a cohort of Finnish patients. J Invest Dermatol. 2000;114(3):587-590.

76. Frentz G, Olsen JH. Malignant tumors and psoriasis: a follow-up study. Br J Dermatol. 1999;140(2):237-242.

77. Chen YJ, Wu CY, Chen TJ, et al. The risk of cancer in patients with psoriasis: A population-based cohort study in Taiwan. J Am Acad Dermatol. 2011;65:84-91.

78. Gelfand JM, Shin DB, Neimann AL, Wang X, Margolis DJ, Troxel AB. The risk of lymphoma in patients with psoriasis. $J$ Invest Dermatol. 2006;126(10):2194-2201.

79. Gupta MA, Gupta AK. Depression and suicidal ideation in dermatology patients with acne, alopecia areata, atopic dermatitis and psoriasis. Br J Dermatol. 1998;139(5):846-850.

80. Gupta MA, Schork NJ, Gupta AK, Kirkby S, Ellis CN. Suicidal ideation in psoriasis. Int J Dermatol. 1993;32(3):188-190.

81. Polenghi MM, Molinari E, Gala C, Guzzi R, Garutti C, Finzi AF. Experience with psoriasis in a psychosomatic dermatology clinic. Acta Derm Venereol Suppl (Stockh). 1994;186:65-66.

82. Choi J, Koo J. Quality of life issues in psoriasis. J Am Acad Dermatol. 2003;49:57-61.

83. Weiss S, Kimball A, Liewehr D, Blauvelt A, Turner M, Emanuel E. Quantifying the harmful effect of psoriasis on health-related quality of life. J Am Acad Dermatol. 2002;47:512-518.

84. Rubino IA, Sonnino A, Pezzarossa B, Ciani N, Bassi R. Personality disorders and psychiatric symptoms in psoriasis. Psychol Rep. 1995; 77(2):547-553.

85. Gupta MA, Gupta AK, Watteel GN. Early onset ( $<40$ years age) psoriasis in comorbid with greater psychopathology than late onset psoriasis: a study of 137 patients. Acta Derm Venereol. 1996;76(6): 464-466.

86. Richards HL, Fortune DG, Weidmann A, Sweeney SK, Griffiths CE. Detection of psychological distress in patients with psoriasis: low consensus between dermatologist and patient. Br J Dermatol. 2004;151(6): 1227-1233.

87. Rapp S, Feldman S, Exum M, Fleischer A, Reboussin D. Psoriasis causes as much disability as other major medical diseases. $J$ Am Acad Dermatol. 1999;41:401-407. 
88. Devrimci-Ozguven H, Kundakci TN, Kumbasar H, Boyvat A. The depression, anxiety, life satisfaction and affective expression levels in psoriasis patients. J Eur Acad Dermatol Venereol. 2000;14(4): 267-271.

89. Rieder E, Tausk F. Psoriasis, a model of dermatologic psychosomatic disease: psychiatric implications and treatments. Int J Dermatol. 2012;51:12-26.
90. Sampogna F, Tabolli S, Abeni D. The impact of changes in clinical severity on psychiatric morbidity in patients with psoriasis: a follow-up study. Br J Dermatol. 2007;157:508-513.

\section{Publish your work in this journal}

Psoriasis: Targets and Therapy is international, peer-reviewed, open access journal focusing on psoriasis, nail psoriasis, psoriatic arthritis and related conditions, identification of therapeutic targets and the optimal use of integrated treatment interventions to achieve improved outcomes and quality of life. Visit http://www.dovepress.com/testimonials.php to read real quotes from published authors. 